\title{
Cinco horas con Mario a teatro: riflessioni traduttive per un adattamento in lingua italiana
}

\author{
Maria Teresa De Pieri \\ Universidad de Udine \\ maria-teresa.depieri@uniud.it
}

Recibido: 25 de octubre de 2013

Aceptado: 30 de enero de 2014

\section{RIASSUNTO}

Il 26 novembre 1979 Cinco horas con Mario di Miguel Delibes approda al Teatro Marquina di Madrid nel suo adattamento per le scene: è l'esordio di un classico, destinato a restare a lungo in cartellone, e che conferma la grande popolarità riscossa nel 1966, quando uscì il romanzo omonimo. Il testo narrativo delibiano presenta, in sé, già alcuni requisiti marcatamente teatrali, come la condensazione della storia e la sua organizzazione formale, ma anche la scarsa rilevanza attribuita al narratore eterodiegetico, praticamente assente, mentre si dà ampio spazio a un "monodialogo" femminile energico e avvincente. L'articolo si propone dunque di analizzare l'incidenza di tali caratteristiche in rapporto a una possibile resa italiana, tenendo presente la necessità di rispettare la verosimiglianza dei dialoghi, la specificità del linguaggio (frequentemente colloquiale), nonché l'autenticità del messaggio autoriale. Si considereranno, infine, alcune delle scelte già operate nel 1983 da Olivo Bin, autore dell'unica versione italiana del romanzo (Cinque ore con Mario), e si valuteranno le differenti operazioni traduttive in rapporto alla specificità della comunicazione narrativa e teatrale.

Palabras clave: Delibes, traduzione teatrale, transcodificazione, monodialogo, colloquialismi.

Cinco horas con Mario on stage:

Some considerations about an adaptation into Italian

\begin{abstract}
On November 26th, 1979, Cinco Horas con Mario was staged at the Marquina Theater in Madrid, as a theatrical adaptation of Miguel Delibes' homonymous novel, first published in 1966. This play was a great success, as the novel was, too, when it was published. In fact, the novel itself has some features that are linked to a theatrical environment: the condensation of the plot, its formal organization, the absence of a heterodiegetic narrator, while, throughout the novel, there is ample room for a capturing and vigorous feminine monologue. This work takes into consideration these features, with refer to a translation of the theatrical


text into Italian, keeping in mind the necessity of respecting the verisimilitude of dialogues, the specificity of the language (often colloquial), and also a correct reading of the message of the author. We shall also analyze some of the choices made in 1983 by Olmo Bin, who translated the novel into Italian, and we shall take into consideration some translational options with reference to the specificity of the comunication that takes place within the book and on stage.

Keywords: Delibes, translating a pièce, theatrical adaptation, monologue, colloquialisms.

Sumario: 1. Introduzione 2. Considerazioni linguistiche e proposte traduttive 3 . Conclusione 4. Appendice.

\section{Introduzione}

Della copiosa produzione letteraria di Miguel Delibes, la versione teatrale del romanzo omonimo, Cinco horas con Mario ${ }^{1}$, è uno degli esiti più felici, nonostante lo scrittore abbia sempre praticato il genere drammatico con incerto entusiasmo: "En el teatro me coarta mucho la limitación de tiempo y la limitación de espacio. Es decir, que lo que ocurre en el drama que tú quieres narrar no tenga más que una hora y media o dos horas de duración. Y otro tanto diríamos del espacio físico: toda tu historia debe estar ceñida a uno, dos o tres escenarios a lo sumo. Estas limitaciones me molestan". ${ }^{2}$ L'economicità propria del mezzo teatrale, a cui egli ricorre sempre per via indiretta (nasce, infatti, prima l'opera narrativa e poi la trasposizione dram-

1 La pièce, prodotta da José Sámano (che curò l'adattamento, assieme allo stesso Delibes, a Santiago Paredes e a Josefina Molina), viene rappresentata per la prima volta al Teatro Marquina di Madrid il 26 novembre 1979, con la regia di Josefina Molina. Il ruolo di Carmen Sotillo viene affidato all'attrice Lola Herrera, che lo interpreterà per circa venticinque anni, in seguito alle successive tournée che ebbero luogo nel 1984 e 1989. Del 2001 è, invece, il secondo adattamento dell'opera, che debutta al Teatro Calderón di Valladolid il 6 ottobre. Attualmente, Cinco horas con Mario continua ad essere presente nei cartelloni teatrali spagnoli, sempre diretta da Molina e interpretata, questa volta, da Natalia Millán. Della versione teatrale esiste anche una traduzione in francese (che purtroppo non ha visto le stampe) di Christine Defoin (1994), andata in scena solo nell'agosto del 2002, durante l'inaugurazione del Festival de Teatro de Spa. L'edizione a stampa del dramma spagnolo si deve, invece, a Gonzalo Sobejano, pubblicata nel 1981 dalla casa editrice Espasa-Calpe di Madrid (n. 83 della collana "Selecciones Austral"); nel 2010 il testo è confluito nel vol. VI delle Obras completas. El periodista. El ensayista. Barcelona: Destino. Studi comparativi tra la versione teatrale e il romanzo sono di: Amorós, A., Herrera, L., Sacristán, J., Ibáñez, M., Narciso y Sámano, J., "De la novela al teatro" (Mesa redonda) in: Miguel Delibes. Premio Letras Españolas 1991. Madrid: Ministerio de Educación, Cultura y Deporte. Secretaría de Estado de Cultura 1994, 259-274; R. M. Tabernero, "Cinco horas con Mario: de la novela al drama", Rilce, III, 1 (1987), che ripercorre sostanzialmente le linee espresse da G. Sobejano; J. R. Valles Calatrava, "Del papel a las tablas: el proceso transductivo de Cinco horas con Mario (novela, versiones, representaciones teatrales)", Rlit, LXVI, 132 (2004), 485-502. Non ci è stato possibile, invece, consultare una tesi di dottorato che affronta la questione con taglio monografico: C. Vega Martín, Novela y teatro en Miguel Delibes: un estudio comparativo. Universidad de Málaga: Servicio de Publicaciones e Intercambio Científico 1996. Cfr. anche S. MARTín, "Tres manifestaciones teatrales: Delibes, Cervantes-Nieva y Alfonso Vallejo", Cuadernos Hispanoamericanos, 363 (1980), 604-614.

2 Miguel DeliBes, "Notas a la edición”, in: Obras completas (2010), vol. VI. El periodista. El ensayista. Barcelona: Destino 1981. 
matica), permette comunque a Miguel Delibes di non sacrificare pressoché nulla dei suoi romanzi, di mantenerne perlopiù inalterati gli elementi strutturali e contenutistici; così avviene per l'adattamento di Cinco horas, in cui permangono l'asciuttezza della trama, l'assenza di un narratore eterodiegetico e il predominio del monologo femminile. Inoltre - ambito che più ci interessa ai fini del presente lavoro - 1'opera narrativa non subisce rettifiche formali di particolare rilievo a seguito dell'operazione di transcodifica, e rimane, anche nella sua nuova veste drammatica, un testo linguisticamente compatto. Lo studio che Gonzalo Sobejano premette all'edizione della pièce, nel 1981, ripercorre puntualmente tagli, riduzioni, modifiche e aggiunte in rapporto al testo-fonte, ma riconosce che il processo di riscrittura non intacca né l'ossatura tematica (sesso, denaro e autorità continuano a prevalere nei discorsi di Carmen) ${ }^{3}$, né tantomeno il taglio formale del romanzo. Lo stile di Delibes, dominato dalla "sobriedad"4 e dalla precisione lessicale, si condensa nel lungo monologo della protagonista, attraverso il quale ella ripercorre la propria vita coniugale con il marito Mario, scomparso da poche ore, rivivendo, di riflesso, i momenti salienti della Spagna franchista. ${ }^{5}$ Il suo argomentare, convenzionale e borghese, costellato da banali cliché, è serrato e massicciamente ripetitivo; è il linguaggio "de lo dicho", come sottolinea Sobejano, una incessante e ossessiva reiterazione di frasi e pensieri che si reggono su un continuo ricorso al registro colloquiale. ${ }^{6}$ Negli inter-

\footnotetext{
3 C. Martín-Gaite, "Sexo y dinero en Cinco horas con Mario" in: Miguel Delibes. Premio Letras Españolas 1991, cit., 131-154.

${ }^{4}$ G. SOBEJANO, "Introducción" alla versione teatrale di Cinco horas con Mario, cit., 15. Per uno studio dettagliato delle caratteristiche stilistiche dell'autore si rimanda a: F. ABAD NeBOT, "Apuntes sobre el estilo y la lengua de Miguel Delibes" in: Miguel Delibes: el escritor, la obra y el lector. Barcelona: Anthropos 1992, 215-224; P. AlCAlÁ ArÉvalo, "El léxico y la evolución estilística de Miguel Delibes en su narrativa" in: Miguel Delibes: el escritor, la obra y el lector, cit., 193-206; A. GIL, "Experimentos lingüísticos de Miguel Delibes" in: Crítica semiológica de textos literarios hispánicos. Actas del Congreso Internacional sobre Semiótica e Hispanismo celebrado en Madrid en los días 20 al 25 de Junio de 1983, a cura di Miguel Angel Garrido Gallardo, Consejo Superior de Investigaciones Científicas 1986, 631-640; S. de los Mozos, "Consideraciones lingüísticas sobre Miguel Delibes”, in: García Domínguez, R. y Santoja, G. (eds.), El autor y su obra: Miguel Delibes. Madrid: Editorial Actas/Universidad Complutense 1993, 89-99. A. Bensoussa, "Un lenguaje pacífico" in: El autor y su obra, cit., 111-118. G. VolKMANN, "La doble deixis como recurso de relativización epistémica en Cinco horas con Mario de Miguel Delibes" in: Deixis y modalidad en textos narrativos, Hassler, G. / Volkmann, G. (eds.). Münster: Nodus 2009, 123-140.

5 Cfr. A. Sotelo Álvarez, Carmen en 'Cinco horas con Mario'de M. Delibes y la mujer española en el franquismo. Vigo: Aristos 1995 e M. L. Bustos Deuso, La mujer en la narrativa de Delibes. Universidad de Valladolid: Secretariado de Publicaciones e Intercambio Editorial 1990.

${ }^{6}$ In merito alla specificità del monologo della protagonista, qualche spunto interessante è fornito da X. A . PAdilla GARcíA, "El análisis conversacional en la obra de Miguel Delibes Cinco horas con Mario" in: Panorama de la investigació lingüística a l'Estat espanyol: Actes del I congrés de lingüística general, a cura di B. Gallardo Paúls, D. Jorques Jiménez, M. A. Alcina Caudet, M. Veyrat Rigat, E. N. Serra Alegre, Vol. 4, Universitat de València 1997, 155-158. Utile è anche l'articolo di A. Gil, "La reproducción literaria del coloquio en la novelística de Miguel Delibes" in: Aspekte der Hispania im 19. Und 20. Jahrhundert, Akten des Deutschen Hispanistentages, Dieter KremER (ed.), Hamburg: Buske 1983, cit., 175-187. Acute, inoltre, le osservazioni di Alfonso Rey, il quale sostiene che: "Llámese monólogo si se desea, el discurso de Carmen tiene todas las características de un diálogo, aunque, al estar su pretendido interlocutor muerto, sólo hay un hablante. Pero [...] el tú tiene un destinatario que no es Carmen, ya que ésta se dirige a Mario como si estuviera presente" in: A. Rey, "Forma y sentido de Cinco horas con Mario" in: Historia y crítica de la literatura española, a cura di F. Rico MANRiQue, Vol. 8, 1999 (“Época contemporánea, 1939-1980”), 448.
} 
venti di Carmen gli enunciati si susseguono senza un ordine apparente, il suo argomentare è franto e incontrollato, tanto che è costretta a ricorrere spesso ad accorgimenti formali (come possono essere i nessi grammaticali: entonces, pero, pues, bueno, o connettivi del tipo: además, aparte, que, incluso), per restituire un minimo di coesione al discorso. Il suo messaggio è ricco di termini dalla connotazione ambigua, frequentemente polisemici, ma anche di formule familiari stereotipate, la cui resa in italiano diventa di non sempre facile risoluzione; 1'ambito della sintassi, sostanzialmente paratattica, presenta casi frequenti di inversioni, cesure, ellissi, mentre gli enunciati non di rado vengono sospesi e recuperati in sequenze successive del testo. Non mancano le imprecisioni formali (come il laísmo ${ }^{7}$ : pp. 784, 785) e il ricorso generalizzato al que polivalente (p. 788), così come la copiosità di avversative, l'anteposizione dell'articolo determinativo dinanzi ai nomi di persona e la presenza di numerosi segnali discorsivi e interiezioni, indici di un'espressività vigorosa e graffiante. ${ }^{8}$

\section{Considerazioni linguistiche e proposte traduttive}

Di fronte alle particolarità strutturali dell'opera teatrale (in cui il testo, come sostiene Susan Bassnett, "è solo un elemento rispetto alla totalità del discorso"") e

${ }^{7}$ Cfr. l'articolo di J. C. Mc InTYRe, "Laísmo in Miguel Delibes Cinco horas con Mario (1966). Background for foreign students trained in 'correct' Spanish", Tejuelo, 5 (2009), 8-24.

${ }^{8}$ Per le caratteristiche proprie dello spagnolo colloquiale, il testo di riferimento a cui ci è appoggiati è quello di A. BRIz GómEZ, El español coloquial: situación y uso. Madrid: Arco/Libros 1996 e il suo più recente articolo "Lo coloquial y lo formal, el eje de la variedad lingüística" in: De moneda nunca usada. Estudios

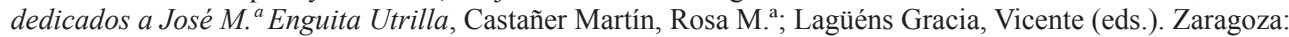
Universidad Autónoma de Nuevo León 2010, 125-133. Cfr. anche, per quanto concerne le specificità formali dei segnali discorsivi, C. Bazzanella, "I segnali discorsivi" in: AA.VV. Grande grammatica italiana di consultazione, Lorenzo Renzi, Giampaolo Salvi e Anna Cardinaletti (eds.). Bologna: Il Mulino 1995, t. III, 225 257, nonché, della stessa autrice, Le facce del parlare. Un approccio pragmatico all'italiano parlato. Scandicci-Firenze: La Nuova Italia 1994; interessante lo studio di Giovanni Nencioni, "Parlato-parlato, parlatoscritto, parlato-recitato", Strumenti critici, 29, fasc. I (1976), 1-56 nonché M. V. Calvi, - G. Mapelli, "Los marcadores 'bueno', 'pues', 'en fin', en los diccionarios de español e italiano”, Artifara, 4 (2004) (www.artifara.com/rivista4/testi/marcadores.asp; consultato il 2/5/2012).

9 S. BAssnett, La traduzione. Teorie e pratica. Milano: Bompiani 1993, 162-163: "Dal momento che una commedia è scritta per delle voci, il testo letterario contiene anche un insieme di sistemi paralinguisti$c i$, in cui l'intensità, l'intonazione, la velocità dell'emissione, l'accento, ecc., sono tutti significanti. Inoltre, il testo teatrale contiene al suo interno un sottotesto o ciò che abbiamo chiamato testo gestuale che determina i movimenti che può fare un attore che reciti quel testo". Indicative, in rapporto alla specificità del codice espressivo teatrale, anche le osservazioni di Serpieri: "[...] un linguaggio che è, sì, letteratura, ma una letteratura tutta particolare: scritta, ma intesa in modo che sia detta e agita, vissuta e rappresentata come mimesi della realtà" (A. SERPIERI, "Intervento" in: AA.VV., La traduzione in scena. Teatro e traduttori a confronto, John Dodds / Ljiljana Avirovic (eds.). Roma: Ministero per i Beni Culturali e Ambientali 1995, 41). Cfr. inoltre l'articolo di M. M. MARTínez-BARTOLOMÉ, "Constraints and Possibilities of Performance Elements in Drama Translation" in: Perspectives, 1 (1995), 21-33, in cui l'autrice riflette sulla complessità semiotica del testo teatrale. Interessante è anche un altro lavoro della medesima autrice, "Translation strategies and the reception of drama performances: a mutual influence" in: Translation as Intercultural Communication. Amsterdam/Philadelphia: Benjamins Translation Library 1997. A pag. 102 si precisa che: "Since the text is 
al taglio stilistico scelto dall'autore, nel momento in cui il traduttore si pone l'obiettivo di una resa in lingua italiana, il suo lavoro dovrebbe preliminarmente tenere presenti alcuni fattori. In primo luogo, la funzione del testo che si accinge a tradurre, ossia stabilire se considerarlo come destinato alla lettura (ragion per cui più opportuna sarebbe la resa letterale e la fedeltà linguistica) o come possibile copione per una futura messa in scena (la cui prioritaria condizione di "recitabilità" potrebbe indurre, invece, anche a significativi adattamenti di ordine linguistico e stilistico). ${ }^{10}$ Inoltre, "la traduzione drammatica", ricorda Serpieri, è "traduzione interlinguistica, come tutte le altre traduzioni, ma anche traduzione delle virtualità intersemiotiche"11, il che richiama l'esigenza di lavorare non solo sulla parola (una "parola" che egli definisce "tridimensionale", vale a dire "legata ad altri sistemi espressivi: il mimico, il gestuale, il prossemico e il cinesico"12), ma anche sugli elementi extratestuali e sulla "funzionalità pragmatica" del discorso. E questa "funzionalità pragmatica" è certamente uno dei caratteri precipui di Cinco horas con Mario, in cui la voce solitaria di Carmen è animata costantemente dalla volontà di "comunicare", ricorrendo ad espedienti, non solo linguistici, che rendono il suo messaggio intensamente dinamico. ${ }^{13}$

La ragione del nostro studio nasce dall'auspicio che anche il pubblico italiano possa, un giorno, confrontarsi con l'intrinseca attualità della pièce. Pur tenendo in debita considerazione quegli elementi contestuali che devono certamente essere adattati all'ambiente della cultura d'arrivo, le scelte traslative compiute sono state guidate essenzialmente da questi obiettivi: la possibile rappresentabilità del testo ${ }^{14} \mathrm{e}$ il rispetto della verosimiglianza e della "pronunciabilità"15 del monodialogo, cer-

only one of the elements of the performance, the translator of plays for performance will have to base his/her translation decisions on a very complex set of factors: the literary and cultural dimension of the text together with the semiotic intricacy of the production and the social characteristics of the target audience".

${ }^{10}$ Cfr. C. Bobes-NAves, "Lengua y literatura en el texto dramático y en el texto narrativo", Bulletin Hispanique, 3-4 (1985), 305-335. Spunti interessanti fornisce, inoltre, S. AALTONEN, Time-Sharing on Stage. Drama Translation in Theatre and Society. Clevedon: Multilingual Matters 2000.

11 SERPIERI, "Intervento", cit., 43.

12 SERPIERI, "Intervento", cit., 44.

13 Cfr. a tal proposito I. Penadés Martínez, "Para un tratamiento lexicográfico de las expresiones fijas irónicas desde la pragmática”, Pragmalingüística, 7 (1999), 185-210.

14 Indicative sono, a questo proposito, le osservazioni di C. Janovic Strada: "Un testo letterario, o non letterario, è tradotto per la lettura $[. .$.$] per una percezione individuale e, per così dire, privata. [...] Diverso$ è il caso di un testo teatrale. [...] la funzione di un testo teatrale è quella di uscire dalla sfera della lettura per entrare in quella della rappresentazione. Un testo teatrale tradotto, dunque, oltre a sottostare ai due momenti della testualità e della contestualità o storicità, è sottoposto al momento della scenicità" (grassetto mio). C. JANOVIC StRADA, "Il teatro di Cechov in italiano. Osservazioni di una traduttrice" in: AA.VV. La traduzione in scena, cit., 257-258.

15 M. M. Martínez-Bartolomé precisa che "la traducción ha de ser pronunciable" [...], mentre qualche passaggio prima aggiunge che [...] "no sólo es importante tener en cuenta lo que los actores pueden hacer, sino también lo que no pueden hacer mientras pronuncian una determinada frase". Inoltre: "[...] el traductor debe apreciar las características fonéticas de los sonidos empleados por el autor: no se trata sólo de palabras con contenido semántico, sino que a menudo la forma fónica de las mismas resulta significativa. Y, lo que es más importante, se ha de tener presente que van a ser pronunciadas por un actor" in: Martínez-Bartolomé, "El componente escénico en la traducción teatral", cit., 912. Il medesimo concetto di "speakability" e "playability" viene espresso da S. Aaltonen, Time-Sharing on Stage, cit., 7. 
cando di rintracciare nella nostra lingua quelle componenti colloquiali che meglio potevano adattarsi al registro informale proprio del testo di partenza. ${ }^{16}$

Le prime osservazioni riguardano l'ambito morfosintattico, in cui si è cercato di ricalcare, nella maggior parte dei casi, il ritmo paratattico dell'enunciazione, anche se, soprattutto per quanto riguarda alcune dislocazioni grammaticali, si è dovuti intervenire adeguando le strutture alle norme dell'italiano. Un chiaro esempio è rappresentato dal dimostrativo "ese" che appare, non solo in Delibes, con un'accezione frequentemente spregiativa ${ }^{17}$ : “[...] es ceguera la de ese hombre y, como él, otros que me callo [...] y tú, que si es un tipo vulgar ese San Juan ..." (p. 779); in questo, come in altri casi, ci è parso che "ese" potesse essere mantenuto attraverso l'elisione di una parte dell'aggettivo e la sostituzione del termine "vulgar" con un lessema più marcato da un punto di vista espressivo, ottenendo una traduzione con " 'sto rozzo". Nell'enunciato "si la bomba atómica esa" (p. 791), l'aggettivo "esa", nuovamente dislocato a destra, si riferisce implicitamente a "la bomba de la que tanto se habla", che potrebbe essere reso di nuovo attraverso il troncamento del dimostrativo, ma con l'anteposizione, questa volta, al sostantivo "bomba", al fine di evitare la ripetizione accessoria data dall'articolo determinativo; inoltre, ci è parso opportuno ricorrere, in questo caso, ad un'ulteriore modifica, aggiungendo l'aggettivo "benedetta", particolarmente efficace dato il tono dell'esclamazione e il contesto all'interno del quale essa è inserita, dando come risultato: "se 'sta benedetta bomba atomica". ${ }^{18}$ Come vedremo, infatti, nonostante la scelta di amplificare l'enunciazione sia sconsigliabile in un testo teatrale, per sua natura conciso, l'operazione si è resa talvolta necessaria per rendere efficacemente alcune battute di Carmen, le quali, nel trasferimento all'italiano, rischiavano di veder sfumare la loro carica illocutoria.

Per quanto concerne la tessitura verbale, nel testo teatrale prevalgono il tempo del presente e, talvolta, quello del "pretérito perfecto", diversamente da quanto accade nell'ipotesto narrativo, dove ad imporsi è piuttosto il "pretérito indefinido": il clima di attualizzazione che si crea con l'avvicinamento temporale è senza dubbio adeguato a un contesto drammatico, e riprende nuovamente una delle costanti proprie del registro colloquiale, in cui l'uso del presente viene indistintamente applicato tanto a eventi passati, come a quelli che ancora devono verificarsi. ${ }^{19} \mathrm{Si}$ è proce-

16 Vanda Durante, nel suo lavoro sulla specificità fraseologica del romanzo di Delibes, sottolinea che è necessario ricreare, nel testo tradotto, l'oralità del linguaggio: "Ello significa che el traductor se preocupará por 'situar pragmáticamente el diálogo' en la lengua de llegada” in: V. DurAnTe, "Modelos translémicos y tratamiento de la fraseología en Cinque ore con Mario", Paremia, 20 (2011), 202.

17 Sull'utilizzo dell'aggettivo con valore spregiativo in Delibes, confronta l'articolo di A. FLORES RAMÍREZ, "Registros lingüísticos e intención comunicativa en El disputado voto del señor Cayo de Miguel Delibes" in: Actas del V Congreso Internacional de Historia de la Lengua Española, Valencia, 31 gennaio - 4 febbraio 2000, a cura di María Teresa Echenique Elizondo, Juan P. SÁnchez MÉndez. Madrid: Gredos 2002, 1171-1190.

18 Anche la traduzione italiana dell'opera narrativa, uscita nel 1983 (Reggio Emilia, Città Armoniosa) e curata da Olivo Bin, propone una resa simile: "[...] se quella benedetta bomba atomica riuscissero a perfezionarla [...]", 145 .

19 Ce lo conferma Gómez BRIz quando sostiene che: “[...] el presente es utilizado como tiempo para actualizar un momento que no concuerda con el momento del habla" in: El español coloquial: situación y uso, cit., 58 . 
duto, dunque, in termini di corrispondenza stilistica anche in questo caso, mantenendo sempre la peculiarità del paradigma verbale scelto da Delibes.

Di carattere informale sono i frequenti segnali discorsivi e le interiezioni: "palabra" (p. 786), abbreviazione di "palabra de honor", è stata resa con un semplice "credimi" (mentre la versione italiana del romanzo - che citeremo a confronto in qualche occasione - rispetta letteralmente il termine, riproducendone, in maniera insistita, la presenza), di certo consono alla voce della protagonista e alla sua relazione intima con l'esanime interlocutore; "fatal" (p. 787), aggettivo ricorrente nello spagnolo orale, meno in quello scritto, che corrisponderebbe all'italiano "tremendo", ma che data la sua collocazione nella frase funziona semplicemente come rafforzativo ("'...] por lo visto, tenía dos hijos con la otra y eso es lo malo, los hijos para estas cosas, según dicen, fatal, difícilismo"); in italiano, l'effetto non può mantenersi traducendo "fatal" per il suo significato letterale, bensì ricorrendo a una intensificazione lessico-semantica data dal raddoppiamento dell'aggettivo "difficile": "difficile, difficilissimo" (Olivo Bin, il traduttore italiano del romanzo, propone invece "non c'è niente da fare, difficilissimo"). Mentre l'originale e categorica "sanseacabó" (p. 777) perde gran parte della propria specificità culturale poiché risulta traducibile solo con un più neutro "non se ne parla", l'interiezione "dale" (p. 787), che spezza volutamente una locuzione figurata esistente in spagnolo ("estar dale que te pego - con algo-, ossia "non smettere neanche un minuto - di fare qualcosa" ${ }^{20}$ ), può essere resa con una interiezione altrettanto agile, "dàgli" (con il significato metaforico di "continui ad insistere"), la quale, in italiano, non rimanda però ad alcun costrutto idiomatico. Altri "marcadores" non hanno invece presentato particolari difficoltà traduttive: è il caso di "por las bravas" (p. 783), "per forza"; "a ver" (pp. 783, 788), "figurati"; "natural", "ovvio" (p. 789), "pero bueno", "però, dài” (p. 791), la cui diffusa presenza contribuisce a potenziare il tono emotivo e ironico delle battute della protagonista.

Numerosi sono anche i lessemi intensificativi, dei quali Carmen si avvale nei momenti di maggiore enfasi comunicativa e che, in alcuni casi, si affievoliscono nel trasferimento all'italiano: quando la protagonista descrive l'incontro fortuito con l'amico Paco, emergono espressioni come "frenazo", "inchiodata" (p. 784), "telefonazo", "colpo di telefono" (p. 797), "cambiazo" (per indicare un cambio eccezionale e inaspettato), traducibile solo ricorrendo a una qualificazione aggettivale: "grande cambiamento" (p. 784). Si tratta di voci che subiscono, attraverso l'alterazione morfologica data dal suffisso, un'espansione semantica rilevante, perché non solo attribuiscono all'evento un'accezione negativa, ma agiscono nel contesto conversazionale come giudizi di valore da parte del parlante, ragion per cui non possono essere in alcun modo trascurati in fase traslativa. ${ }^{21}$ Non manca nemmeno la catego-

\footnotetext{
${ }^{20}$ Cfr. p. 792 del testo teatrale di Delibes, in cui Carmen ribadisce: "Pero tú, dale que te pego [...]". Frequente è anche la perifrasi: "venga de" (p. 800), variazione di "venga a", per trasmettere l'idea di insistenza.

${ }^{21}$ Per maggiori dettagli relativamente alla funzione degli intensificatori nel discorso colloquiale, imprescindibile è il rimando a A. BRIz GóMEZ, El español coloquial en la conversación. Esbozo de pragmagramática. Barcelona: Ariel 1998 (cap. V: "Estrategias de producción- recepción: la intensificación en la conversación coloquial”, 113-142). In: Cinco horas con Mario Delibes ricorre spesso anche a unità fraseologi- 
ria dei diminutivi"22, a cui spesso Delibes attribuisce una funzione parodica: "chiquitín", "piccolino" (p. 777), "igualito que dormido", trasferibile in italiano solo con "come se stesse dormendo" (p. 777); "guapín", "bellino" (p. 795); "curitas", "pretini" (p. 794), "asquito", "schifezzuola" (p. 794), "mismito" (p. 801, diminutivo che, dato il contesto, funziona come un rafforzativo), ancora una volta intraducibile letteralmente in italiano se non con "stessa identica cosa" (p. 801). ${ }^{23}$

A livello lessicale, le tracce del registro informale si ritrovano ovunque; riportiamo alcuni esempi significativi, con la nostra proposta di traduzione, mentre raccogliamo nell'appendice del presente lavoro l'elenco dettagliato di tutte le voci colloquiali e la loro corrispondenza in italiano. È interessante notare come la maggior parte di esse siano semanticamente caratterizzate, di nuovo, in termini negativi: così vale per "querindonga" (con terminazione inusuale in spagnolo) e corrispondente all'italiano "ganzo", "amante" (termine fortemente spregiativo) o "mollas" (p. 795), per indicare i rotolini di ciccia (lessema che oggigiorno pare sia stato diffusamente sostituito con "michelines", rimandando al pupazzo della Michelin); "zalema" (p. 797) "riverenza, ossequio", versione colloquiale di "zalamería" (lusinga, salamelecco); "pepla" (p. 799), per indicare "schifo, schifezza", con una limitata frequenza d'uso in ambito spagnolo. Carmen si rivolge al marito defunto apostrofandolo con epiteti di varia natura ${ }^{24}$ : Mario è, perciò, indistintamente "vejestorio" (p. 779), "vecchio rimbambito" (con evidente tono spregiativo) e "zascandil" (pp. 783, 792), che i dizionari bilingue individuano come "malandrino", ma che risulta decisamente inadeguato in rapporto all'asprezza verbale assunta da Carmen (più consono è, per esempio, "delinquente", o un più generico "canaglia" 25 ). E ancora: "alcornoque" ( $p$. 786), letteralmente "quercia da sughero", ma che in senso figurato indica una "persona ignorante", uno "zuccone". L'aggettivo italiano "sfacciato" viene espresso da Carmen ricorrendo a ben tre colloquialismi differenti, ossia "carota" (p. 786), "fre-

che che sottolineano questa idea di intensificazione semantica: un esempio è il colloquialismo "no tener perdón de Dios" (p. 802), corrispondente all'italiano "essere imperdonabile, gridare vendetta"; "ni pintada" (p. 792), formula sospesa che si utilizza molto in Spagna, per esprimere "alla grande, da dio" o "neanche per sogno".

${ }^{22}$ Una classificazione delle varie modalità di attenuazione linguistica è contenuta sempre in BRIZ GóMEZ, El español coloquial en la conversación. Esbozo de pragmagramática, cit. (cap. VI: "Estrategias de producción- recepción: la atenuación en la conversación coloquial”, 143-163).

${ }^{23}$ Come risulta evidente, al monologo non viene attribuita solo una funzione espressiva, bensì anche fatica e conativa; la traduzione proposta ha tenuto dunque in debita considerazione anche il complesso sistema di segni paralinguistici e prossemici, che in un testo drammatico svolgono sempre una funzione altamente significante. Un articolo interessante sugli appellativi colloquiali in Cinco horas con Mario è di C. Araceli Martínez AlBARRACín, "Los apelativos coloquiales en cuatro novelas españolas de posguerra (C.J. Cela, La Colmena; M. Delibes, Cinco horas con Mario; C.M. Gaite, Entre visillos; R. Sánchez Ferlosio, El Jarama)" in: Actas del II Congreso Internacional de Historia de la Lengua Española, II. Madrid: Pabellón de España 1992, 739-752.

${ }^{24}$ Un articolo interessante sugli appellativi colloquiali in Cinco horas con Mario è di C. Araceli MARTíneZ AlBarRacín, "Los apelativos coloquiales en cuatro novelas españolas de posguerra (C.J. Cela, La Colmena; M. Delibes, Cinco horas con Mario; C.M. Gaite, Entre visillos; R. Sánchez Ferlosio, El Jarama)" in: Actas del II Congreso Internacional de Historia de la Lengua Española, II. Madrid: Pabellón de España 1992, 739-752.

${ }_{25}$ Bin ricorre al termine "pelandrone", che tuttavia ci pare inadeguato perché la sequenza non è intesa, in questo caso, a sottolineare un'ipotetica scioperatezza di Mario, bensì la sua scelta di non scendere a compromessi con i potenti. 
sco" (pp. 788 e 790, con accezione spregiativa) e "cuajo" (p. 794), letteralmente "cuaglio" del latte, ma che nel linguaggio colloquiale indica "flemma", "lentezza". Di contro, gli aggettivi che la protagonista si autoattribuisce appaiono indubbiamente meno ingiuriosi, ma vengono comunque sempre attinti dal brillante repertorio informale: "tarabilla" (p. 793), voce onomatopeica che sta ad indicare un vaniloquio alacre e indiscreto, trasferibile all'italiano con "chiacchierona, parolaia"; "reventada" (p. 779) e "frita" (p. 782), ossia "spompata", "stanca morta", sono i termini che Carmen utilizza per sottolineare la propria abnegazione domestica. Di non trascurabile frequenza anche le voci verbali di matrice colloquiale, che riportiamo in appendice (come per esempio "armar" (p. 783), col significato di "causare, provocare"; "escupir", "sputare, confessare" (p. 784); "pillar", "beccare" (p. 785); "tirar", "attirare" (p. 785); "fisgar", "curiosare, ficcare il naso" (p. 796); "chiflar", "piacere da matti" (p. 796); "pegar", "incollare" (p. 799). Qualche sporadico volgarismo ${ }^{26}$ presente nel testo trova, poi, una corrispondenza pressoché letterale nella nostra lingua: "fulana" (p. 785), equivale a "puttana"; l'aggettivo spregiativo "patán", così come "paleto" (p. 791), si riferisce invece a "campagnolo", "zotico".

Il corpus fraseologico, consistente ed eterogeneo, è senza dubbio l'ambito che più impegna il lavoro del traduttore, non solo nella fase di identificazione e interpretazione delle unità presenti nel testo, ma anche nel momento della ricerca di corrispondenze adeguate. ${ }^{27}$ Molte di queste locuzioni incontrano, nel testo d'arrivo, una equivalenza lessicale e morfosintattica completa (o quasi). Ne diamo una breve sintesi: "tirar del carro" (p. 777) corrisponde all'italiano "tirare la carretta"; "dejar (o poner) en evidencia" (p. 789), "mettere in ridicolo"; "hacerse cargo de algo" (p. 802), "farsi carico di" o "rendersi conto di qualcosa"; "hablar en clave" (p. 803), "parlare in codice, cifrato"; "dar grima" (p. 786), "dar fastidio"; "venir a pelo" (p. 791), "venire a puntino". Di natura informale è la locuzione "importar un bledo"28 (p. 793), "importare un accidenti" o la più comune "llover sobre mojado" (p. 799), "piovere sul bagnato".

26 Sull'utilizzo dei volgarismi in Delibes è uscito di recente un lavoro di G. BRANDIMONTE, "Breve estudio contrastivo fraseológico y paremiológico: El disputado voto del señor Cayo y su versión italiana", Paremia, 20 (2011), 77-87.

27 Ci riferiamo, in questo caso, a quelle unità fraseologiche che, per funzionare all'interno di un discorso, raggiungono una completezza semantica solo appoggiandosi ad altre unità presenti della frase; si distinguono, dunque, dagli enunciati fraseologici, che vedremo più avanti. Gli studi teorici di riferimento consultati sono: C. NAVArro, Aspectos de fraseología contrastiva. Verona: Fiorini 2008; e di G. CoRPAS PASTOR, Manual de fraseología española. Gredos: Madrid 1996 e Diez años de investigación en fraseología. Análisis sintáctico-semánticos, contrastivos y traductológicos. Madrid-Frankfurt: Iberoamericana-Vervuert 2003, in particolare i capp. XI, XIII e XIV. Vedi anche l'articolo di P. ZAMORA MuÑOZ, "Equivalencias lingüísticas de algunas frases idiomáticas pragmáticas italianas en español”, Paremia, 8 (1999), 529-535. In rapporto ai fraseologismi in Delibes, vedi anche Jorge URDIALES YusTE, "Refranes, sentencias, modismos y dichos estereotipados en Cinco horas con Mario", de Miguel Delibes, Revista de folklore, 334 (2008), 141-144. Di recentissima pubblicazione è, invece, il testo di Luciano LóPEZ GuTiÉRrEZ, En torno a las palabras de Delibes, Valladolid, Castilla Ediciones, 2013. Uno studio comparativo tra le possibili corrispondenze fraseologiche, in italiano, dell'opera delibiana è contenuto, inoltre, in: Luis LuQUE Toro, "La equivalencia fraseológica entre español e italiano en Cinco horas con Mario de Miguel Delibes" in: AA. VV., Entre Friuli y Espa$\tilde{n} a$, a cura di R. Londero. Venezia: Mazzanti 2008, 89-95.

28 Si tratta di una locuzione verbale idiomatica e lessicalizzata, che Briz include tra le costruzioni di intensificazione espressiva (El español coloquial en la conversación, cit., 121). 
Un gruppo consistente di costrutti idiomatici sono riproducibili nella nostra lingua attraverso un recupero parziale dei loro costituenti: di natura colloquiale risultano "no quitar(le) ojo a alguien" (p. 800), "non togliere gli occhi di dosso a qualcuno"; "(quedarse) de (una) piedra" (p. 800), abitualmente utilizzato, nella lingua spagnola, senza l'articolo indeterminativo, e che indica in italiano "rimanere di stucco"; "estar en la luna" (p. 787), variazione di "estar en Babia" o "estar en las nubes", per "essere tra le nuvole"; "sacar(le) con sacacorchos algo a alguien" (p. 798), "tirar fuori le parole di bocca col cavatappi a qualcuno"; "bajar el color" (p. 775), che richiede un aggiustamento verbale in italiano, "perdere colore"; "con segundas" (p. 790), "con secondi fini"; "medias tintas" (p. 790), "mezzi termini"; "dejar(le) a alguien con la miel en los labios" (p. 784), "lasciare qualcuno a bocca asciutta". "Estar hecho de otro barro" (p. 791) figura come un'alterazione voluta dall'autore, poiché in spagnolo è decisamente più comune l'espressione "estar hecho de otra pasta", omologo all'italiano "essere fatto di un'altra pasta"; lo stesso vale per la combinazione "agarrar el sueño" (p. 798), in cui Delibes opta per un verbo più energico, mentre la locuzione abitualmente diffusa è, come nella nostra lingua, "prendere sonno" ossia "coger el sueño". ${ }^{29}$

Fra le locuzioni che permettono, nella lingua italiana, di mantenere certamente il medesimo significato, ma che divergono tanto nel lessico quanto nella struttura, gli esempi da citare sarebbero davvero molti; ne ripercorriamo solo alcuni per sottolineare, ancora una volta, come il regime linguistico dato dalla compatta colloquialità del testo sia fortemente vincolante ai fini di una traduzione efficacemente comunicativa, soprattutto se destinata alle scene: è il caso di "entrar pocos en kilo" (p. 774), poco comune anche nello spagnolo attuale ("essercene pochi"); "tener más conchas que un galápago" (p. 777), per "saperla lunga" o "saperne una più del diavolo"; "salir por peteneras" (p. 777), in cui il riferimento culturale al genere musicale andaluso si perde completamente nell'italiano "partire per la tangente"; "irse a las vistillas" (pp. 777, 786), trasferibile con un generico "sbirciare" e, per rimanere nella medesima sfera tematica, "no darle a alguien vela en (o para) un entierro" (p. 793), equivalente a "non permettere a qualcuno di ficcare il naso". Per riferirsi alla stupidità del marito, Carmen sfodera anche una lunga serie di varianti fraseologiche: "ser tonto de capirote" (p. 781), per "essere completamente scemo", equivalente all'alternativa "ser tonto del bote" ${ }^{30}$, nonché a "ser tonto de baba" (p. 799), "essere scemo col botto". Sono, poi, le azioni che hanno attinenza con l'ambito comunicativo e relazionale quelle che vengono più frequentemente trattate attraverso $\mathrm{i}$ fraseologismi: per "criticare aspramente qualcuno" Carmen ricorre alla locuzione "poner de vuelta y media a alguien" (p. 782), mentre quando agisce in termini opposti, ossia per "adulare qualcuno", efficace le risulta il "bailar(le) a alguien el agua"

29 Cfr. BRIz, El español coloquial en la conversación, cit., 25-26.

30 Dizionari di riferimento sono: DEA= Manuel Seco, Olimpia Andrés, Gabino Ramos, Diccionario del español actual. Madrid: Aguilar 1999, 2 tt.; Manuel Seco, Nuevo diccionario de dudas y dificultades. Madrid: Espasa-Calpe 2011; DFEA= Manuel SECO, Olimpia AndRÉs, Gabino RAmos, Diccionario fraseológico documentado del español actual. Locuciones y modismos españoles. Madrid: Aguilar 2004. 
o il più indecoroso "darle vaselina a alguien" (p. 781, "indorare la pillola"). Infine, Paco, l'amico "segreto" della protagonista, la esorta a non curarsi delle malelingue, dicendole "déjales que digan misa" (p. 800), vale a dire "mi entra da un orecchio e mi esce dall'altro". Un piacevole gioco fonico creano alcune voci onomatopeiche: "dar(le) un patatús" (p. 786) capita quando a qualcuno gli "viene un colpo", mentre "el año patapum" (p. 803), ulteriore variazione delibiana per "catapún" (p. 803), potrebbe essere reso con una locuzione metaforica equivalente, "ai tempi di Noè", ma in cui viene a perdersi del tutto la sonorità della frase. ${ }^{31}$ Simbolica e fonica insieme è l'ulteriore "no decir ni pío" (p. 792) per segnalare l'incapacità di proferire parola, in cui valida riformulazione, stilistica e semantica, starebbe nell'italiano "non dire né a né ba".

Rispetto alla modalità traduttiva degli enunciati fraseologici ${ }^{32}$, si è cercato di mediare tra la consonanza letterale e una rielaborazione più libera, pur rimanendo fedeli al terreno concettuale del testo-fonte: “¿A Santo de qué?”33 (p. 782, 797), data la natura colloquiale del testo, ammetterebbe una traduzione con "Perché diavolo?", ma si è preferita una resa più generica, "Perché poi? Per quale motivo?", poiché le imprecazioni puritane di Carmen mai si appellano alla figura dell'antagonista di Cristo. "¡Vaya un trago, hijo!" (p. 792), è stato reso con "Che momentaccio!" (mentre il romanzo nella sua traduzione italiana propone "cosa non ho passato", p. 83); "Punto en boca" 34 (p. 797) risulta trasferibile solo con "Punto e basta!", fra l'altro già glossato dal dizionario bilingue, ma non riconosciuto da Bin, che erroneamente lo rende con "acqua in bocca" (p. 179), per nulla logico rispetto al contesto della sequenza ${ }^{35}$; infine, "Sólo faltaría", "Ci mancherebbe altro!" (p. 777), unico caso in cui la traduzione del romanzo concorda con quella da noi proposta per il teatro (p. 188).

L'opera narrativa sfoggia, infine, un patrimonio paremiologico davvero sorprendente, mentre la versione drammatica lo riduce ad alcuni significativi campioni ${ }^{36}$ : "En la vida vale más una buena amistad que una carrera" ${ }^{37}$ (p. 782), viene tradotto letteral-

$31 \mathrm{Nel}$ romanzo l'espressione appare in più occasioni, ma il traduttore italiano la restituisce con esiti spesso diversi: “chissà quale anno" (265) oppure "nell'anno del Signore" (133 e 170).

32 Ricordiamo che, a differenza delle locuzioni figurate, gli enunciati fraseologici (tra i quali vanno inclusi anche i proverbi) sono sequenze linguistiche indipendenti, che funzionano in maniera autonoma all'interno di un testo.

33 Seco precisa che della locuzione avverbiale esiste una variante più rara, “A qué Santo?”, corrispondente all'italiano "Per quale motivo?", con chiara intenzione polemica in: DFEA, cit., 912.

34 Seco sottolinea, infatti, che questa formula colloquiale viene utilizzata "para proponer una actitud, volontaria o impuesta, de silencio" in: DFEA, cit., 846.

35 "Desde aquí te digo que tendría más vista, que las tontas somos nosotras por vivir pendientes de los maridos y de los hijos, que a Valen la sobra razón, que se adelanta más no mostrando excesivo interés, lógico, que, si no, cogéis y ¡hala!, a exigir, tráeme esto y lo otro y lo de más allá, que os lo creéis todo debido los hombres, todos cortados por el mismo patrón, Mario, por más que lo tuyo pase ya de castaño oscuro, que con los extraños venga zalemas y atenciones y en casa, punto en boca, que eso es lo que peor llevo, fíjate" in: Cinco horas con Mario, cit., 237.

${ }^{36}$ L'unico articolo che analizza il tema in questione è quello di J. PIETRZAK, "Cinco horas con Mario de Miguel Delibes: estudio traductológico de los refranes en la edición polaca y alemana”, Paremia, 20 (2011), 53-64.

37 Gran parte dei proverbi presenti nel testo vengono formulati indirettamente da Carmen, poiché sono generalmente attribuibili alla saggezza popolare della madre di lei; in effetti, ad introdurli è spesso 
mente da Bin con "Gli amici [...] possono valere più di una carriera" (p. 65), quando invece pare più giustificato, anche in rapporto alla frequenza d'uso in ambito italiano: "Un amico vale più di un tesoro". Per "Casarse con un primo hermano o con un hombre de clase inferior es hacer oposiciones a la desgracia" ${ }^{38}$ (p. 782), Bin propone: "Maritarsi con un primo cugino o con un uomo di classe inferiore è come volersi tirare addosso la disgrazia" (p. 72). Sevilla Muñoz, nel suo studio comparativo sui "refranes" spagnoli ${ }^{39}$, propone, a nostro parere, una soluzione poco ricorrente in italiano: "Il parentato dev'esser pari” (p. 82); ci è parso dunque pertinente indirizzarci verso una formula più colloquiale: "Sposarsi con un primo cugino o con un uomo di classe inferiore significa volersi tirare la zappa sui piedi". Per la successiva "El que no llora no mama" (p. 783), concordiamo con Bin, il quale propone: "Chi non piange il morto non pappa" (p. 245), mentre nell'alternativa proposta da Sevilla Muñoz, "Chi non piange, non pappa" (p. 139), il carattere figurato del verbo "piangere" si smarrisce completamente. "Per una donna la verginità è la maggior virtù" è la traduzione che è stata scelta per "Para una mujer la pureza es la prenda más apreciada" (p. 786), mentre ci è parsa poco efficace la versione proposta da Bin: "Per una donna la purezza è il gioiello più prezioso" (p. 177), una raffinatezza espressiva che mal si armonizza con lo stile, talvolta prosaico, di Carmen. Infine, con "A la vejez viruelas" (p. 795) ha colto bene Bin traducendo il proverbio con uno corrispondente in italiano, "ogni frutto alla sua stagione" (p. 185 e p. 204), sicuramente diffuso e normalmente utilizzato nella lingua d'arrivo. ${ }^{40}$

\section{Conclusione}

Molti aspetti dell'opera di Delibes analizzati in questa sede hanno un carattere necessariamente esemplificativo. Ci è sembrata comunque una valida occasione per riflettere non solo sulla ricchezza espressiva della lingua spagnola (e ci riferiamo soprattutto alla vivacità stilistica del registro colloquiale, di cui Cinco horas con Mario costituisce una straordinaria sintesi), ma anche per ritornare su alcune problematiche inerenti il lavoro del traduttore. L'articolato discorso di Carmen ha richiesto, come abbiamo visto, interventi significativi nel trasferimento alla lingua d'arrivo, perdendo in molti casi - specialmente nella sua impalcatura fraseologica - il vigore idiomatico e simbolico. Le acute riflessioni di Serpieri ${ }^{41}$ ci hanno indirizzati a sce-

l’espressione "como diría la pobre mamá”; "mamá siempre decía”; “Sabes lo que decía mamá a este respecto?". Per quanto concerne questa prima paremia, essa appare, nel romanzo, con una struttura differente: "Y los amigos, ya lo decía la pobre mamá, que en paz descanse, pueden valer más que una carrera", 128. Proponiamo anche la soluzione scelta dalla traduttrice francese dell'opera teatrale, Christine Defoin, "Bonne amitié vaut mieux que bon métier".

38 La locuzione "hacer oposiciones a algo" significa, in spagnolo, "cercarsi qualcosa", il che giustifica la scelta di Bin per "volersi tirare addosso" (qualcosa). La versione francese della Defoin riporta: "Ma fille, épouser son cousin germain ou un homme de classe inférieure c'est prendre une option sur le malheur".

39 J. Sevilla Muñoz / J. Cantera Ortiz de Urbina, 1001 refranes españoles. Madrid: Ediciones Internacionales Universitarias (2001) 2008.

40 Defoin propone, per la lingua francese, "ça lui a pris sur le tard".

41 Serpieri afferma, infatti, che: "[...] il testo transcodificato, tradotto, inevitabilmente tradito, deve solo cercare di rimanere denso come l'originale, anche a costo di risultare talvolta ostico alla lettura. I grumi di 
gliere questa strada: a tradurre e a transcodificare il testo, conservandone tanto la compattezza formale, quanto le sporadiche "oscurità". Risulta sorprendente il contrasto tra il linguaggio colloquiale di Carmen e il suo ideale di vita, non certo popolare, ma dietro questa palese e ironica discordanza Miguel Delibes forse ha voluto comunicarci altro. Un "altro" che, probabilmente, troverà la sua piena realizzazione, anche linguistica, solo a teatro, e solo nell'intima esegesi di ogni spettatore. ${ }^{42}$

\section{Appendice:}

\section{I: locuzioni fraseologiche}

A carta cabal (p. 783): al cento per cento

A ciencia y paciencia (p. 793): (colloq.) coscienziosamente

A lo tonto (p. 796): (colloq.) senza accorgersene, senza rendersene conto

A nadie le amarga un dulce (p. 784): (colloq.) nessuno rifiuta una cosa gradevole

Abrírsele a alguien las carnes (p. 781): (colloq.) raggelarsi il sangue a qualcuno

Agarrar el sueño (p. 798): (colloq.) prendere sonno
Ir a pata/ita (p. 779): (vulg. colloq.) andare a piedi/ni

Irse a las vistillas (p. 777, 786): (colloq.) sbirciare

Llover sobre mojado (p. 799): (colloq.) piovere sul bagnato

Manga por hombro (p. 787): (colloq.) sottosopra

Medias tintas (p. 790): (fig.) mezzi termini

Ni entrar ni salir (p. 782): (colloq.) non intervenire

senso si chiariranno nell'attivazione della parola dentro i codici scenici. Mimare le opacità della scrittura drammatica, che diventano poi punti di forza sulla scena, significa riprodurre il sistema del testo originale. Se, invece, ci si lascia tentare da parafrasi, perifrasi, aggiunte, scioglimenti, per risolvere quelle opacità già sul piano della lettura, si potrà chiarire qualche segmento, ma si infrangerà il sistema e si effettuerà una sottrazione di teatralità. [...] Il traduttore è destinatario mentre decodifica e interpreta il testo, ma diventa destinatore quando lo codifica nuovamente nella sua lingua: è lettore e autore, sia pure di secondo grado. Ma è essenziale che egli non si comporti "da lettore" anche nell'atto della scrittura. Un eccesso di "lettura" in questo senso rischia di diluire e quindi tradire il testo originale, proprio mentre può essere inteso a rendergli giustizia esplicitandone le complessità e le oscurità. [...] Il pericolo dell'eccesso di lettura, e quindi di spiegazione parafrastica, da parte del traduttore, riguarda tutte le traduzioni, ma è ancor più marcato nella traduzione drammatica, perché, come già detto, nel dramma il significato potenziale, implicito, segreto, si affida anche, e forse soprattutto, alla convocazione più o meno dichiarata dei codici scenici che ne devono completare ed esaltare il senso" in: "Intervento", cit., 41-42.

${ }_{42}$ M. Grazia Profeti ricorda che "Ogni messa in scena è traduzione, traduzione nel linguaggio scenico del testo letterario per il teatro, passibile (nella serie delle sue potenzialità) di essere detto in dispositivi scenici diversi, anche cambiando in maniera decisiva - attraverso le modalità della comunicazione - il messaggio stesso offerto dal testo". M. G. Profeti, "Lope de Vega in Italia. Le traduzioni, la fortuna" in: AA.VV., La traduzione in scena, cit., 135. Clara Janovic parla di "traduzione complementare" consistente nella "teatralizzazione" del testo, "cioè nell'opera degli attori, registi, costumisti, scenografi. Traduzione, questa seconda, forse ancor più difficile della prima, puramente linguistica. E può accadere che il traduttore, che ha volto il testo teatrale $[\ldots]$ in un'altra lingua e il cui testo sia stato adottato da un teatro, non riconosca il "suo" [...]". "Il teatro di Cechov in italiano. Osservazioni di una traduttrice" in: AA.VV., La traduzione in scena, cit., 260. Infine, Marta Mateo sottolinea che: "the study of drama translation should not only include the written text with all the features that make it performable but also individual performances in which the text will have acquired its total experience" in: MARTíNEZBARTOlOMÉ, "Constraints and Possibilities of Performance Elements in Drama Translation", cit., 24. 
Bailar(le) a alguien el agua (p. 783): adulare qualcuno

Bajar el color (p. 775): perdere colore

Como si dice (o que diga) misa (p. 800): (colloq.) mi entra da un orecchio e mi esce dall'altro, può dire quello che vuole

Como un cohete (p. 800): (colloq.) come un razzo

Con segundas (p. 790): con secondi fini

Dar grima (p. 786): dar fastidio, far venire i brividi, far senso

Dar la vuelta a la tortilla (p. 790): (colloq.) invertire, rovesciare (sorte, situazione, ruoli)

Dar un patatús (p. 786): venire un colpo a qualcuno

Darle (o ponerle) vaselina a alguien (p. 781): (colloq.) indorare la pillola, lisciare il pelo

De pata de banco (p. 794): (colloq.) gaffe

De vicio (p. 797): (colloq.) senza motivo, per abitudine; stupendamente, meravigliosamente

Decir a boca llena (p. 794): (colloq.) dire papale papale, esplicitamente

Dejar (o poner) en evidencia (p. 789): mettere in evidenza; mettere in ridicolo

Dejar(le) a alguien con la miel en los labios (p. 784): lasciare qualcuno a bocca asciutta

Echar chispas (p. 802): (colloq.) fare fuoco e fiamme, dare in escandescenze (essere infuriato)

El año patapum (o catapún) (p. 803): ai tempi di Noè

En plan de (p. 801): (colloq.) con l'intenzione di

(En un) visto y no visto (p. 801): (colloq.) (in un batter d'occhio)

Encogerse de hombros (p. 803): alzare le spalle

Entrar pocos en kilo (p. 774): essercene pochi

Estar como una balsa de aceite (p. 791): (colloq.) tranquillo (molto)

Estar en la luna (p. 787): (colloq.) essere tra le nuvole

Estar forrado (p. 784): essere strapieno di soldi

Estar hecho de otro barro (p. 791): essere fatto di un'altra pasta

Estar hecho un asco (p. 796): (colloq.) essere ridotto da schifo

Hablar en clave (p. 803): parlare in codice, cifrato

Hablar en plata (p. 787): (colloq.) parlare senza giri di parole

Hacer ascos a algo (p. 785, ma anche prima): fare lo schizzinoso
Ni pintada (p. 792): (colloq.) alla grande, da dio, neanche per sogno No dar ni frío ni calor (p. 799): (colloq.) non fare né caldo né freddo

No darle a alguien vela en (o para) un entierro (p. 793): (colloq.) non permettere a qualcuno di ficcare il naso

No decir ni pío (p. 792): (colloq.) non dire né a né ba

No dejar a alguien ni a sol ni a sombra (p. 779): (colloq.) seguire qualcuno come un'ombra

No estar en sus cabales (p. 794, 801): (colloq.) essere fuori di testa

No haber (no tener) vuelta de hoja (p. 799): (colloq.) non esserci dubbio

No quitar(le) ojo a alguien (p. 800): (colloq.) non togliere gli occhi di dosso a qualcuno

No sentir ni padecer (p. 783): essere (o mostrarsi) insensibile

No tener perdón de Dios (p. 802): (colloq.) essere imperdonabile, gridare vendetta

Pasar de castaño oscuro (p. 797): (colloq.) oltrepassare ogni limite

Pasar la rueda de Santa Catalina (p. 784): tirare troppo la corda (sp. tirar de la cuerda)

Poner de vuelta y media a alguien (p. 782): (colloq.) criticare aspramente qualcuno

Poner patas arriba (p. 790): mettere sottosopra, a rovescio

Punto en boca (p. 797): (colloq.) punto e basta

Quedarse a solas (p. 776): rimanere soli

Quedarse de un aire (p. 801): rimanere stupito

(Quedarse) de (una) piedra (p. 800): (colloq.) (rimanere) di stucco

Revolver Roma con Santiago (p. 789): mettere tutto sottosopra

Sacar de quicio a alguien (p. 787, 791): far uscire qualcuno dai gangheri

Sacar(le) con sacacorchos algo a alguien (p. 798): (colloq.) tirar fuori le parole di bocca col cavatappi a qualcuno

Salir por peteneras (p. 777): (colloq.) partire per la tangente

Sentir en el alma (p. 788): dispiacersi di cuore

Ser tonto de capirote (p. 781): (colloq.) essere completamente scemo

(Ser tonto) de baba (bote) (p. 799): (colloq.) (essere scemo) di brutto, essere scemo col botto

Tener más conchas que un galápago (p. 777): (colloq.) saperla lunga 
Hacerse a la idea (p. 803): abituarsi all'idea

Hacerse cargo de algo (p. 802): farsi carico di; rendersi conto di qualcosa

Hacerse un ovillo (p. 776): raggomitolarsi

Importar un bledo (p. 793): (colloq.) importare un accidenti

Ir (o pasar o llegar) a mayores (p. 785): oltrepassare il limite, aggravarsi
Tener un detalle con alguien (p. 792): avere un pensiero gentile verso qlcu.

Tirar del carro (p. 777): tirare la carretta

Tirar por la borda (p. 781): (colloq.) tirare alle ortiche

Venir a pelo (p. 791): (colloq.) far comodo, venire a puntino

Venir con cuentos (p. 801): (colloq.) raccontare balle

\section{II: enunciati fraseologici}

- ¿A Santo de qué? (p. 782, 797): (colloq.) Perché poi? Per quale motivo?

- Punto en boca (p. 797): (colloq.) Punto e basta!

- "Sólo faltaría", Ci mancherebbe altro! (p. 777)

-iVaya un trago, hijo! (p. 792): (colloq. fig.) Che momentaccio!

\section{III: voci colloquiali}

\section{Sostantivi:}

Alcornoque (p. 786): (fig.) ignorante, zuccone Aperreada (p. 778): duro, snervante (vita da cani)

Bola (p. 795): frottola, palla

Cabezonada (p. 780): cocciutaggine

Carota (p. 786): sfacciato

Cuajo (p. 794): (fig.) flemma

Dichoso (p. 799): 1 fortunato, felice 2 maledetto, dannato

Fresco (p. 788, 790): (spreg.) sfacciato

Frita (p. 782): morta (stanca morta)

Golpe (p. 785): trovata

Guasa (p. 785): ironia

Latoso (p. 782): noioso, palloso

Mollas (p. 795): rotolini di ciccia

Nena (p. 796): pupa, bimba; tesoro (affet.)

Papelote (p. 779): cartaccia, cartastraccia

\section{$\underline{\text { Verbi: }}$}

Armar (p. 783): causare, provocare

Chiflar (p. 796): piacere da matti

Cascar (p. 789): ridurre male; pestare; spettegolare; crepare

Colarse (p. 796): (colloq.) 1 intrufolarsi 2 prendersi una cotta (por alguien) 3 sbagliarsi
Parranda (p. 777): baldoria (estar de parranda $=$ fare baldoria)

Pataleta (p. 782): scenata, sfuriata

Pelaje (p. 778): aspetto

Pepla (p. 799): schifo, schifezza

Querindonga (p. 775): gonza, amante (spreg.)

Reventada (p. 779): spompata (stanca morta)

(San)seacabó (p. 777): basta, non si discute

Sofocón (p. 792): colpo (di caldo, di calore)

Tarabilla (p. 793): chiacchierona, parolaia

Telefonazo (p. 797): colpo di telefono, squillo

Tronchante (p. 788): spassoso

Vejestorio (p. 779): (spreg.) vecchio rimbambito, vecchio bacucco

Zalema (p. 797): riverenza, ossequio

Zascandil (p. 783, 792): malandrino

Escupir (p. 784): sputare, confessare

Fisgar (p. 796): curiosare, ficcare il naso

Pegar (p. 799): incollare

Pillar (p. 785): beccare

Tirar (p. 785): attirare 


\section{IV: Paremie}

- En la vida vale más una buena amistad que una carrera (p. 782): Un amico vale più di un tesoro

- Casarse con un primo hermano o con un hombre de clase inferiore es hacer oposiciones a la desgracia (p. 782): Sposarsi con un primo cugino o con un uomo di classe inferiore significa volersi tirare la zappa sui piedi

- El que no llora no mama (p. 783): Chi non piange il morto non pappa

- Para una mujer la pureza es la prenda más apreciada (p. 786): Per una donna la verginità è la maggior virtù

- A la vejez viruelas (p. 795): ogni frutto alla sua stagione

\section{Riferimenti bibliografici}

Aaltonen, S., Time-Sharing on Stage. Drama Translation in Theatre and Society. Clevedon: Multilingual Matters 2000.

Abad Nebot, F., «Apuntes sobre el estilo y la lengua de Miguel Delibes», in: Miguel Delibes: el escritor, la obra y el lector. Barcelona: Anthropos 1992, 215-224.

Alcalá ArÉvalo, P., «El léxico y la evolución estilística de Miguel Delibes en su narrativa», in: Miguel Delibes: el escritor, la obra y el lector, cit., 193-206.

Amorós, A. / Herrera, L. / Sacristán, J. / IbÁÑez, M. / Narciso y SÁmano, J., «De la novela al teatro» (Mesa redonda) in: Miguel Delibes. Premio Letras Españolas 1991. Madrid: Ministerio de Educación, Cultura y Deporte. Secretaría de Estado de Cultura 1994, 259-274.

Bassnett, S., La traduzione. Teorie e pratica. Milano: Bompiani 1993.

Bazzanella, C., «I segnali discorsivi» in: Renzi, L. / Salvi, G. / Cardinaletti, A. (eds.), Grande grammatica italiana di consultazione. Bologna: Il Mulino 1995, t. III, 225-257.

- Le facce del parlare. Un approccio pragmatico all'italiano parlato. Scandicci-Firenze: La Nuova Italia 1994.

Bensoussa, A., «Un lenguaje pacífico» in: García Domínguez, R. / Santoja, G. (eds.), El autor y su obra. Madrid: Universidad Complutense 1993, 111-118.

Bobes-NAVEs, C., «Lengua y literatura en el texto dramático y en el texto narrativo», Bulletin Hispanique 3-4 (1985), 305-335.

Briz GómeZ, A., El español coloquial: situación y uso. Madrid: Arco/Libros 1996.

- El español coloquial en la conversación. Esbozo de pragmagramática. Barcelona: Ariel 1998.

- «Lo coloquial y lo formal, el eje de la variedad lingüística», in: CASTAÑER MARTín, Rosa M. ${ }^{a}$ / LAGÜÉNS GRACIA, Vicente (eds.), De moneda nunca usada. Estudios dedicados a José M. ${ }^{a}$ Enguita Utrilla. Zaragoza: Universidad Autónoma de Nuevo León 2010, 125-133.

CABrera, V., "Los lectores ficticios y metaficticios de 'Cinco horas con Mario'» (http://tell.fll.purdue.edu/RLA-Archive/1995/Spanish html /Cabrera, Vicente. htm, consultato il 27/4/2012)

Calvi, M. V. / Mapelli, G., «Los marcadores 'bueno', 'pues', ‘en fin', en los diccionarios de español e italiano», Artifara 4 (2004) (www.artifara.com/rivista4/testi/marcadores.asp; consultato il 2/5/2012).

CARbonell, S., Dizionario fraseologico completo italiano spagnolo e spagnolo-italiano. Milano: Hoepli [1950-1953] 1983. 
Corpas Pastor, G., Manual de fraseología española. Madrid: Gredos 1996.

- Diez años de investigación en fraseología. Análisis sintáctico-semánticos, contrastivos y traductológicos. Iberoamericana-Vervuert: Madrid-Frankfurt 2003.

DeliBes, M., Cinco horas con Mario (1966). Barcelona: Destino 2010.

- Cinque ore con Mario. Traduzione di Olivo Bin. Reggio Emilia: Città armoniosa 1983.

- «Breve reflexión sobre mi obra literaria», in: KREMER, D. (ed.), Aspekte der Hispania im 19. Und 20. Jahrhundert. Akten des Deutschen Hispanistentages, Hamburg: Buske 1983, 165-174.

DEA= Seco, Manuel - Andrés, Olimpia - Ramos, Gabino, Diccionario del español actual. Madrid: Aguilar 1999, 2 tt. (2011).

SECO, M., Nuevo diccionario de dudad y dificultades. Madrid: Espasa-Calpe 2011.

Dethier-Rongé, M., "Sobre unos problemas de traducción” in: Actas de XI Congreso de la Asociación Internacional de Hispanistas. Berlino, 18-23 agosto 1986. Coord. Sebastián Neumeister. Vol. 1 (1989), 103-112.

Devoto, G. / Oli, G. C., Il dizionario della lingua italiana. Firenze: Le Monnier 2000.

DFEA= SECo, M. / Andrés, O. / RAMos, G., Diccionario fraseológico documentado del español actual. Locuciones y modismos españoles. Madrid: Aguilar 2004.

DíAz-Plaja, G., «Cinco horas con Mario de Miguel Delibes», ABC, 16 febbraio 1967.

$\mathrm{DRAE}=$ Diccionario de la Real Academia. Madrid: Real Academia de la Lengua $\left(21^{\mathrm{a}}\right.$ edizione) 1992.

DUE= Moliner, M., Diccionario de uso del español. Madrid: Gredos 1990, 2 tt. (consultabile anche in internet).

Durante, V., «Modelos translémicos y tratamiento de la fraseología en Cinque ore con Mario», Paremia 20 (2011), 201-210.

Fages, G. C., «Subversión de los roles sociales y de género en Cinco horas con Mario», Tonos 15 (2008).

FAINI, P., Tradurre. Dalla teoria alla pratica. Roma: Carocci 2004.

FERrero, J., «Lectura de Cinco horas con Mario» in: Miguel Delibes: homenaje académico y literario, cit., 180-186.

Flores RAMíreZ, A., «Registros lingüísticos e intención comunicativa en El disputado voto del señor Cayo de Miguel Delibes» in: Actas del V Congreso Internacional de Historia de la Lengua Española, Valencia, 31 gennaio - 4 febbraio 2000, a cura di María Teresa ECHENIQue Elizondo e Juan P. SÁnCHEZ MéndeZ. Madrid: Gredos 2002, 1171-1190.

Florin, S., «Realia in translation», in: Zlateva, P. (ed.), Translation as Social Action. Russian and Bulgarian Perspectives, London: Routledge 1993, 122-128.

Fuente, DE LA, B., «La lengua como medio de opresión en la novelística de Miguel Delibes» in: Aspekte der Hispania im 19. Und 20. Jahrhundert, cit., pp. 189-202.

García-PosadA, M., «Cinco horas con Mario: una revisión» in: Cuevas García, C. (ed.), Miguel Delibes. El escritor, la obra y el lector, cit., 115-129.

GIL, A., «La reprodución literaria del coloquio en la novelística de Miguel Delibes» in: Aspekte der Hispania im 19. Und 20. Jahrhundert, cit., pp. 175-187.

- «Experimentos lingüísticos de Miguel Delibes» in: Crítica semiológica de textos literarios hispánicos. Actas del Congreso Internacional sobre Semiótica e Hispanismo celebrado en Madrid en los días 20 al 25 de Junio de 1983, a cura di Miguel Angel GARRIDo Gallardo, Consejo Superior de Investigaciones Científicas 1986, 631-640.

Guerrero, O., «Miguel Delibes y su novela Cinco horas con Mario», Cuadernos Hispanoamericanos 210 (1967), 614-621.

Larraz, F., «Aspectos ideológicos en Cinco horas con Mario de Miguel Delibes», Revista chilena de literatura 74 (2009), 213-223. 
LuQue TORO, L., «La equivalencia fraseológica entre español e italiano en Cinco horas con Mario de Miguel Delibes» in: AA. VV., Entre Friuli y España, a cura di Renata LonDERO. Venezia: Mazzanti 2008, 89-95.

Manzo Robledo, F., «Aspectos formales e ideológicos en la exploración de la conciencia femenina de Cinco horas con Mario (1966), de Miguel Delibes», Espéculo 14 (2000).

Martínez-BARTOlomé, M. M., «El componente escénico en la traducción teatral» in: M. Edo Juliá (ed.), Actes del I Congrés Internacional sobre Traducció, Universitat Autònoma de Barcelona, Bellaterra 1992, 907-917.

- «Translation strategies and the reception of drama performances: a mutual influence» in: Translation as Intercultural Communication. Amsterdam / Philadelphia: Benjamins Translation Library 1997, 99-111.

- «Constraints and Possibilities of Performance Elements in Drama Translation», Perspectives 1 (1995), 21-33.

Martín-Gaite, C., «Sexo y dinero en Cinco horas con Mario» in: Miguel Delibes. Premio Letras Españolas 1991, cit., 131-154.

Martín, S., "Tres manifestaciones teatrales: Delibes, Cervantes-Nieva y Alfonso Vallejo», Cuadernos Hispanoamericanos 363 (1980), 604-614.

Martínez Albarracín, C. A., «Los apelativos coloquiales en cuatro novelas españolas de posguerra (C.J. Cela, La Colmena; M. Delibes, Cinco horas con Mario; C. M. Gaite, Entre visillos; R. Sánchez Ferlosio, El Jarama)» in: Actas del II Congreso Internacional de Historia de la Lengua Española, II. Madrid: Pabellón de España 1992, 739-752.

Mc Intyre, J. C., «Laísmo in Miguel Delibes Cinco horas con Mario (1966). Background for foreign students trained in 'correct' Spanish», Tejuelo 5 (2009), 8-24.

Mozos, DE LOS, S., «Consideraciones lingüísticas sobre Miguel Delibes» in: GARCíA DomínGUEZ, Ramón y SAntoja, Gonzalo (eds.), El autor y su obra: Miguel Delibes, cit., 89-99.

Nencioni, G., «Parlato-parlato, parlato-scritto, parlato-recitato», Strumenti critici 29, I (1976), 1-56.

PIETRZaK, J., "Cinco horas con Mario de Miguel Delibes: estudio traductológico de los refranes en la edición polaca y alemana», Paremia 20 (2011), 53-64.

Quartu, B. M., Dizionario dei modi di dire della lingua italiana. Milano: BUR 1993.

REY, A., «Forma y sentido de Cinco horas con Mario», in: Historia y crítica de la literatura española, a cura di Francisco Rico MANRIQUE, Vol. 8, 1999 («Época contemporánea, 1939-1980»), 448-454.

Scelfo Micci, M. G., Aspetti della traduzione letteraria: riflessioni in margine alla versione italiana di un romanzo di M. Delibes. Napoli: Istituto Universitario Studi Orientali 1984, 249-274.

— «Tradurre 1' 'altro': tra ideologia e manipolazione», Quaderni sulla traduzione letteraria XXXV, 135 (supplemento n. 10) (2002), 5-14.

SERPIERI, A., «Intervento», in: AA.VV., La traduzione in scena. Teatro e traduttori a confronto. John DodDs e Ljiljana Avirovic (eds.). Roma: Ministero per i Beni Culturali e Ambientali 1995.

Sevilla Muñoz, J. / Cantera Ortiz de Urbina, J., 1001 refranes españoles. Madrid: Ediciones Internacionales Universitarias (2001) 2008.

Sevilla Muñoz, J. / Zurdo, M. ${ }^{\mathrm{a}}$ T., Refranero multilingüe. Centro Virtual Cervantes, http://cvc.cervantes.es/lengua/refranero, 2009.

SobejANO, G., «Introducción» alla versione teatrale di Cinco horas con Mario. Madrid: Espasa-Calpe 1981.

— «Los poderes de Antonia Quijana (sobre Cinco horas con Mario de Miguel Delibes)». Alicante: Biblioteca Virtual Miguel de Cervantes 2009. 
TABernero, R. M., «Cinco horas con Mario: de la novela al drama», Rilce III, 1 (1987).

TAM, L., Grande Dizionario di Spagnolo. Spagnolo-italiano/italiano-spagnolo. Milano: Hoepli [1997] 2004.

URdiales Yuste, J., «Refranes, sentencias, modismos y dichos estereotipados en Cinco horas con Mario, de Miguel Delibes», Revista de folklore 334 (2008), 141-144.

Valles Calatrava, J. R., «Del papel a las tablas: el proceso transductivo de Cinco horas con Mario (novela, versiones, representaciones teatrales)», Rlit LXVI, 132 (2004), 485502.

ViLAnova, Antonio, «Introducción» in: Miguel Delibes, Cinco horas con Mario. Barcelona: Destino 1996.

- «Cinco horas con Mario o el arte de entender las razones del otro», in: Cuevas García, C. (ed.), Miguel Delibes, El escritor, la obra y el lector, cit., 131-193.

ZAMORA MuÑoz, P., Spagnolo-italiano: espressioni idiomatiche e proverbi. Milano: EGEA 1997.

— «Equivalencias lingüísticas de algunas frases idiomáticas pragmáticas italianas en español», Paremia 8 (1999), 529-535.

Zingarelli, N., Il Nuovo Zingarelli - Vocabolario della lingua italiana. Bologna: Zanichelli 1983 . 